\title{
A FRAMEWORK COUPLING VISSIM AND OMNET++ TO SIMULATE FUTURE INTELLIGENT TRANSPORTATION SYSTEMS
}

\author{
Tibor Petrov ${ }^{1, *}$, Ilya Finkelberg ${ }^{2}$, Nina Zarkhin², Peter Počta ${ }^{3}$, Luboš Buzna ${ }^{1,4}$, Ayelet Gal-Tzur², Tatiana Kováčiková1, \\ Tomer Toledo ${ }^{2}$, Milan Dado ${ }^{3}$ \\ ${ }^{1}$ Department of International Research Projects - ERAdiate+, University of Zilina, Zilina, Slovakia \\ ${ }^{2}$ Transportation Research Institute, Technion - Israel Institute of Technology, Haifa, Israel \\ ${ }^{3}$ Department of Multimedia and Information - Communication Technologies, Faculty of Electrical Engineering and \\ Information Technology, University of Zilina, Zilina, Slovakia \\ ${ }^{4}$ Department of Mathematical Methods and Operations Research, Faculty of Management Science and Informatics, \\ University of Zilina, Zilina, Slovakia \\ *E-mail of corresponding author: tibor.petrov@uniza.sk
}

\section{Resume}

With the spread of connected vehicles (CVs), a growth of novel information services exploiting data transmitted by CVs is expected. Wireless communication systems, in particular in vehicular applications, operate with a varying level of transmission reliability, which may affect the quality of V2X-data-driven intelligent transport systems (ITS). Therefore, the performance of ITS should be evaluated in a variety of conditions and the configuration of parameters should be fine-tuned in a safe testbed, using computer simulations. A simple framework is presented, which couples VISSIM traffic simulation and OMNeT++ communication networks simulation in real time, enabling an assessment of the relationship between a communication reliability and transport service quality. A functionality of the framework is demonstrated by applying it to a scheme controlling signalized intersections while estimating traffic flows from the V2I data.

Available online: https://doi.org/10.26552/com.C.2021.2.C23-C29

\section{Article info}

Received 30 June 2020

Accepted 10 August 2020

Online 15 January 2021

\section{Keywords:}

connected vehicles,

traffic control,

communications reliability,

simulation framework

ISSN 1335-4205 (print version)

ISSN 2585-7878 (online version)

\section{Introduction}

As the population grows, so does the road congestion in urban areas and this trend is expected to continue in the near future. One of the means to counter the increased delays at the bottlenecks of urban network - signalized intersections, is to develop more advanced and efficient signal control algorithms utilizing emerging technologies. One of the promising near future solutions is the utilization of traffic data from connected vehicles (CVs) for a more advanced and efficient traffic control. Contrary to today's common practice, where approaching vehicles are detected by sensors at fixed locations, the Vehicle to Infrastructure (V2I) communications allow the intersection controller to receive richer and more precise traffic data (e.g. current location, speed and direction of travel) [1] for every connected vehicle, allowing a more advanced and efficient intersection signal control. A large variety of control algorithms and methods are being developed [2], where the connected vehicle data is used as an input for the decision making process and where the main distinction between the proposed algorithms is the specific green light allocation scheduling technique and the used objective function.
The proposed techniques rely heavily on an assumption of perfect communication (i.e. no latency and message loss), however, in the real world applications the reliability of communication networks might be affected by various factors [3-4]. Several studies investigate communication networks performance in connected vehicles environment [5-6] and while the effect of information transmission quality on vehicles' routing decisions in a signalized urban network has been investigated [7], the direct, real-time impact of imperfect communication on traffic control decisions at signalized intersections is less studied. As a field test validation of various traffic scenarios in connected vehicle environment might be too expensive and complex to set up, a common practice in traffic control research is to create simulation models. In this study, a simulation framework that integrates VISSIM traffic micro-simulation software and OMNET++ communication networks simulator was built in order to provide a testbed for realistic interaction scenarios. In the future work, the developed test bed/framework may provide some valuable insights into an impact of imperfect communication on, for example, signal traffic control performance in connected vehicles environment and possible ways to decrease the 
sensitivity of the control algorithm to communication faults, or warning applications such as notification of the CV drivers about an approaching emergency vehicle [8]

\subsection{Traffic simulation platform}

VISSIM [9], a state-of-the-art traffic microsimulation software and preferred platform for evaluation of connected vehicle environment scenarios, is used in this study. The software package comes with advanced traffic behavioral models, a large variety of customization options and a built-in API (Application Programming Interface) module, which makes VISSIM a good choice for advanced traffic control applications. The COM (Component Object Model) and API interface provide a powerful development tool to simulate a connected vehicle environment control with vehicle-infrastructure communication, where the simulation of communication network performance at each step is carried out by an external program.

\subsection{Communication network simulation platform}

Current communication technologies have gradually evolved into mathematically complex and highly dynamic systems [10]. Hence, the easiest and most precise way of evaluating their performance and behavior, besides conducting field measurements, is running computer simulations. Among many simulation engines available, the Objective Modular Network Testbed in $\mathrm{C}++(\mathrm{OMNeT}++)$ has gained extensive popularity due to its scalability, efficient memory usage and wide range of available simulation models [11]. Instead of being a simulator of a concrete aspect of communication networks, it rather provides infrastructure and tools for writing simulations [12]. The generic architecture of OMNeT++ therefore enables simulation of various problem domains, e.g. wired/wireless networks, communication protocols, queuing networks and many more [13]. This versatility and modularity of its architecture led the community to develop a wide range of open-source simulation modules and model libraries for $\mathrm{OMNeT}_{++}$, including the most extensive one - INET framework [14]. The INET supports a variety of communication networks, including wired, wireless, mobile, ad hoc and sensor networks. It contains models for the Internet stack, application modules, routing protocols, link layer protocols and physical layer models among many other components. The INET also provides a support for node mobility and serves as a base or as an extension to other simulation frameworks extending OMNeT++ towards a specific direction, such as Veins [15], designed particularly for simulating vehicular networks.

\subsection{Federated telco-traffic simulators}

The simulation of vehicular communications implies meeting of specific requirements on the side of the communication network simulator. Besides the support of the vehicular communication protocols, which are different than in the case of conventional networks, it also has to be able to mimic vehicle mobility to model movements of the communication network nodes. To achieve realistic vehicular-like mobility in communication network simulation, an output from a traffic simulation is needed. This is a complex problem, since the position of a particular vehicle in the next simulation step can often depend on the result of previous data transmissions in the communication network. Hence, both simulators need to be coupled and perform a bi-directional synchronization.

Such a simulation framework - Veins - has been in development since 2006 [7]. The Veins bi-directionally couples the OMNeT++ with Simulation of Urban Mobility (SUMO) [16], an open-source microscopic traffic simulator. Moreover, Veins package provides simulation models of vehicular communication protocols, messages, nodes and sample applications, all compliant with IEEE WAVE family of standards [17]

However, many municipalities, transport planning experts and researchers around the world prefer using the VISSIM for traffic simulation, which, compared to the SUMO, has easier network coding, built-in ability to model public transport and pedestrians, GIS support and 3D visualization [18]. As a result, the variety of realistic and calibrated test cases available in VISSIM format is much higher. If these existing traffic simulation models are to be extended to employ the CVs, there is no straightforward way to convert them into a format compatible with the SUMO without the expense of extensive manual modifications. Hence, the Veins framework cannot be used for simulating these models without their complete and time-consuming rebuild in the SUMO.

To address the above-mentioned issue, a simulation framework is proposed, which integrates the VISSIM and OMNeT++ to allow simulations involving the CVs in both traffic and telecommunications domains.

\section{Framework description}

A simulation utilizing the proposed framework includes one or more pairs of the following elements:

- VISSIM entity - a model of an element occurring in the traffic simulation that is expected to have communication capabilities, i.e. a connected vehicle, a pedestrian exploiting V2P communication, etc. Each such component is defined, among other parameters, by a unique VISSIM ID and its instantaneous position;

- $\quad$ OMNeT++ node - a model of an element occurring in the communication network simulated by the $\mathrm{OMNeT}++$ simulator. Each $\mathrm{OMNeT}++$ node represents a single coupled VISSIM entity present in the traffic simulation.

Architecture of the framework containing developed simulator extension modules and flows of data is depicted in Figure 1. 


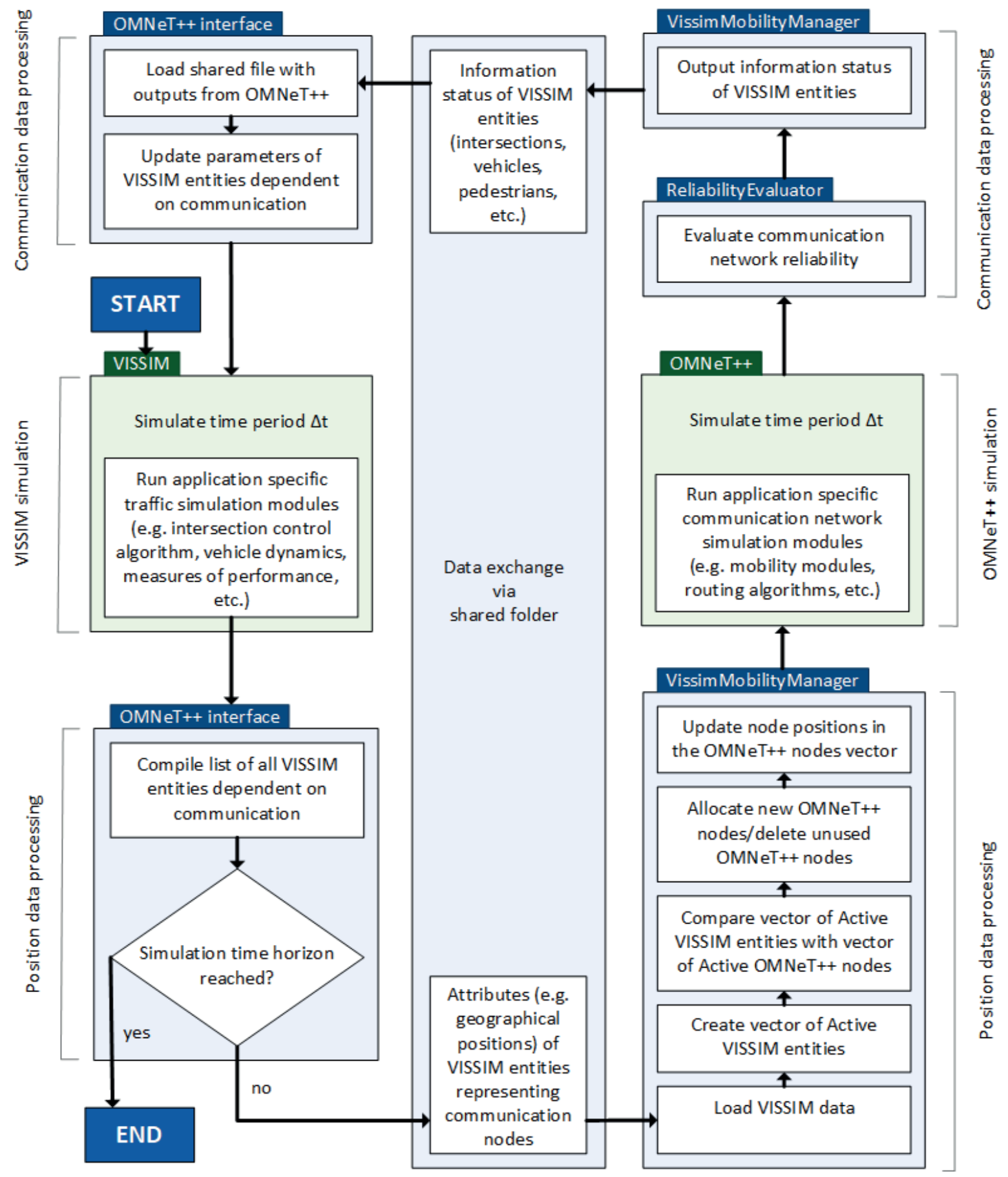

Figure 1 Architecture of the VISSIM-OMNeT++ simulation framework

The framework consists of two separate interfaces (implemented in VISSIM and OMNeT++), which exchange data via a shared folder. The VISSIM's interface (see Figure 1 - the left-hand side) controls updating of the VISSIM entities' parameters, based on their communication status received from the $\mathrm{OMNeT}++$ in the previous simulation period $\Delta \mathrm{t}_{\mathrm{i}-1}$. The VISSIM subsequently runs the specific traffic simulation modules responsible for implementation of the traffic control algorithms used in the simulation, as well as for generation of the VISSIM entities' position updates. After simulating current period $\Delta t_{i}$ with updated parameters of the VISSIM entities, interface compiles a list of all the VISSIM entities and their attributes for simulation step $\Delta t_{i}$, and writes them to the shared file. Afterwards, the interface suspends the simulation in the VISSIM until a shared file containing communication network simulation output from the OMNeT++ is created.

The interface implemented in the OMNeT++ (see Figure 1 - the right-hand side) consists of three modules, which execute the framework's communication network simulation management:

- VissimMobilityManager - a core module of the 
OMNeT++ part of the framework. It is responsible for managing positions of all the OMNeT++ nodes, writing an output of communication network simulation and maintaining synchronization with the VISSIM;

- VissimMobility - a mobility module, where each OMNeT++ node has to take part in the federated simulation. It is responsible for updating node's position and visual representation in the OMNeT++ GUI;

- Communication network ReliabilityEvaluator - a module responsible for collecting and storing statistics about communication reliability parameters, e.g. number of sent and received messages, average message loss, end-to-end delay, etc.

A shared file containing the VISSIM IDs of all the communicating entities and their position in the current simulation step is generated as an output. This file is loaded by the VissimMobilityManager module. The OMNeT++ nodes are managed by the two main data structures in the VissimMobilityManager, i.e. Active VISSIM Entities (AVE) vector and Active OMNeT++ Nodes (AON) vector. The AVE vector contains the IDs of all the VISSIM entities present in the simulation within the current simulation period $\Delta t_{i}$. The AON vector contains IDs of all the active OMNeT++ nodes present in the simulation within $\Delta t_{i}$, as well as their positions and mapping to the IDs of VISSIM entities.

After loading the VISSIM entity data from the shared file, the AVE vector is initialized. The AVE vector is subsequently compared to the AON vector containing entries from the previous simulation period $\Delta$ ti-1. For each new VISSIM entity in the AVE vector, an object in the AON vector is created and OMNeT++ node is assigned. The AON entries not present in the AVE are then destroyed along with the OMNeT++ nodes they represent. In the next step, the position of all the objects present in the AON vector is updated. After that, the positions of the OMNeT++ nodes are updated from the AON vector by rewriting previous coordinates in each OMNeT++ node's VissimMobility module.

Once the positions of all the OMNeT++ nodes are updated, the OMNeT++ proceeds with a simulation of data transmissions in the communication network. During this period, the corresponding OMNeT++ modules log target output parameters to the ReliabilityEvaluator and VissimMobilityManager modules. At the end of the current simulation period $\Delta t_{i}$, the VissimMobilityManager creates a shared file with communication status of each VISSIM entity and halts the OMNeT++ simulation until a next shared file with the input from the VISSIM for a simulation period $\Delta t_{i+1}$ is created and stored in the shared directory.

\section{Demonstration of the framework functionality and applicability}

To showcase the framework functionality and applicability, a demonstration scenario, involving a dense network of the CVs broadcasting messages to a Roadside Unit (RSU), was implemented. A four-leg intersection was simulated, which is controlled by an adaptive traffic control algorithm utilizing data from connected vehicles received by the RSU.

\subsection{Traffic Signal Control Algorithm}

The traffic control algorithm relies on received input data from the CVs, thus the performance of the communication network is expected to have an impact on the traffic control efficiency. Each vehicle in the vicinity of the intersection serves as a traffic detector by transmitting its speed, location and planned route to the intersection controller via Vehicle-To-Infrastructure communication channel. The signal control algorithm, which represents an extension of the existing work [19], uses the received data to assign a weight coefficient to every CV. A coefficient value is calculated as a function of vehicles' distance from the stopline, where the closer vehicles receive higher values and higher priority in a green light allocation process. Given each vehicle's planned route, the approaching CVs are then grouped into Signal Groups (SGs) that will service them. At the next step, the SGs are combined into Signal Phases (SPs), i.e. the combinations of Signal Groups that can receive green light simultaneously. By this type of aggregation, using vehicles coefficients, each SP is assigned a score, which has a direct relationship with its priority level in the green light allocation process. As the minor extension of the original work [19], a waiting time constraint was implemented, i.e. all the SGs with non-zero demand need to be serviced before starting the next signal cycle. In addition, the SP scores from the current cycle influence the maximum green duration in the next cycle, providing more green duration if necessary for the SPs with a higher demand.

\subsection{Communication network simulation}

In the simulation, the RSU antenna was located on a traffic light pole, 5.897 meters above the ground (corresponding to the top of the traffic lights) to ensure a line-of-sight communication. Each vehicle was equipped with an IEEE 802.11p [20] compliant communication module and broadcasted messages with a $1 \mathrm{~Hz}$ frequency. To simulate a challenging communication environment, an electromagnetic interference was modelled by setting the communication channel's background noise power to -86 $\mathrm{dBm}$. A path loss of the wireless signal was modeled by the Two-Ray Interference model [21].

For the visualization purpose, the framework alters vehicle colors according to their communication status (operational/non-operational) in the current simulation time period $\Delta t$. The RSU successfully received messages from vehicles visualized in black color, while the information from the yellow vehicles was not available to the RSU at the corresponding simulation time period (see Figure 2 for illustration). 


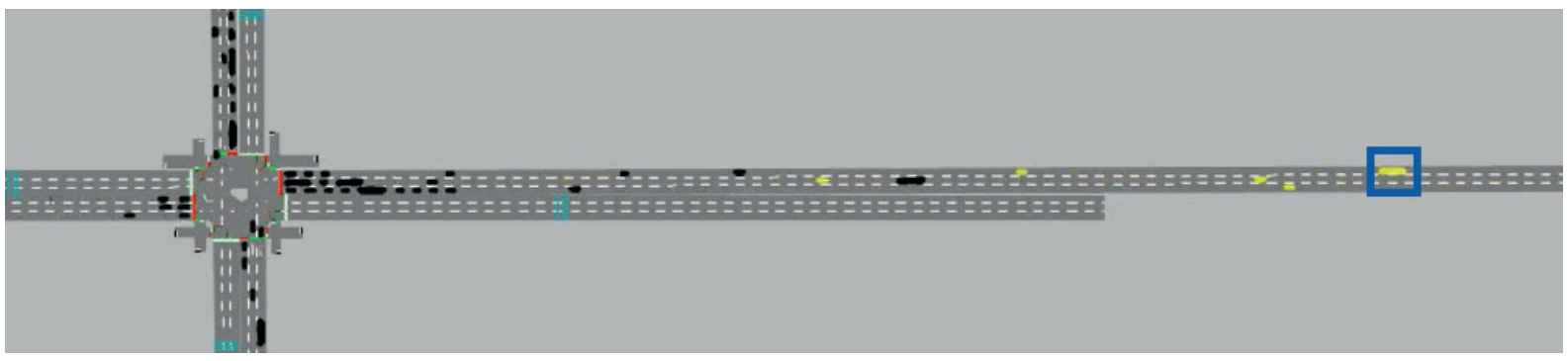

(a)

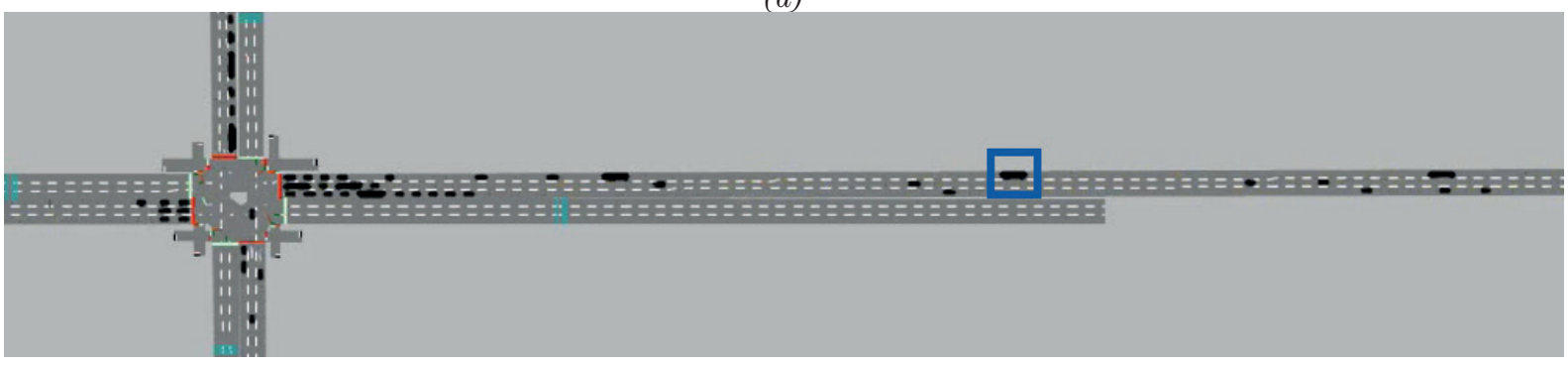

(b)

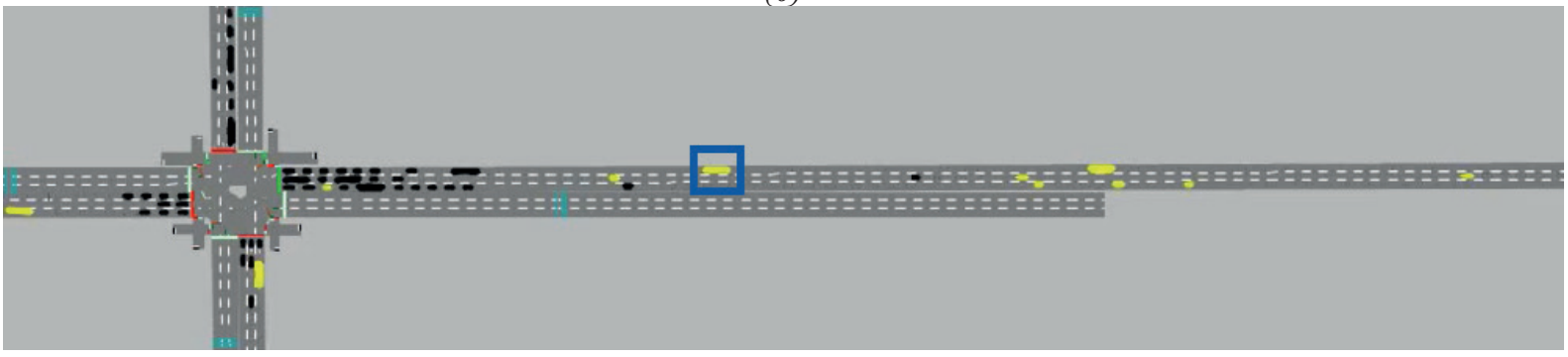

(c)

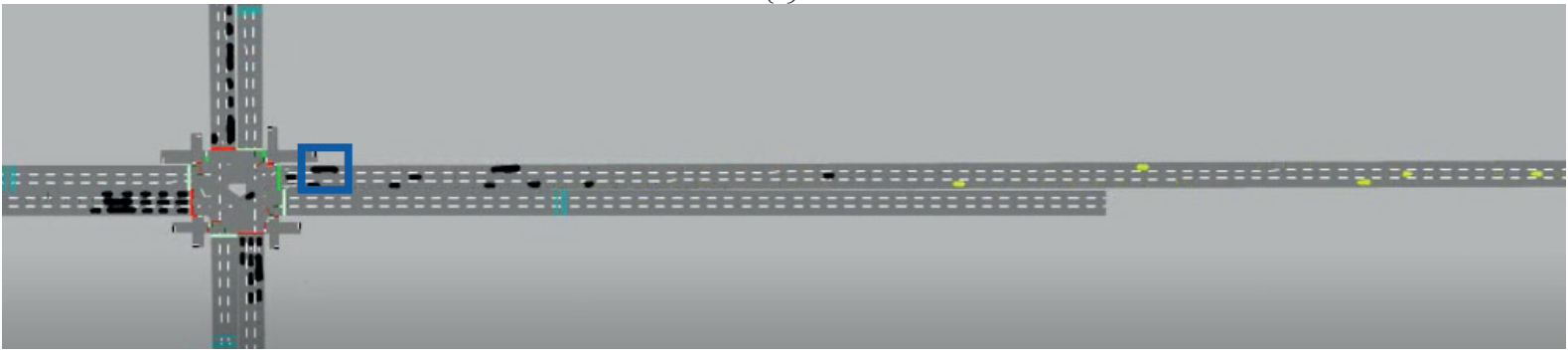

(d)

Figure 2 Communication status of a selected vehicle (a public transport bus in the blue square) during the simulation: (a) communication with the RSU is faulty due to an RF signal distortion caused by electromagnetic interference; (b) the bus has successfully sent a message to the RSU; (c) a message from the vehicle was lost due to the hidden node problem [22]; (d) the vehicle is close enough to the RSU to communicate successfully despite the relatively high level of interference

The communication errors are more likely to occur with the increasing distance between the CVs and the RSU due to the combined effects of electromagnetic interference and signal attenuation. As the same communication channel is shared by all the vehicles, errors can also occur at the closer ranges due to a co-channel interference between transmissions from other vehicles (Figure 2c). The communication error rate achieves its minimum in the close vicinity of the RSU. Here, even when the communication channel is noisy, the vehicles are still able to transmit the data/information successfully. It is worth noting here that the information transmitted by the CVs can be utilized to optimize the green allocation process in order to decrease queueing delays.

\section{Conclusions and discussion}

To facilitate the use of existing calibrated traffic models implemented in the VISSIM for CV simulations, a simulation framework was proposed and implemented, coupling the VISSIM traffic simulator and OMNeT++ communication networks simulator. The framework supports a real-time bidirectional communication between both simulators, as well as a visualization of the CVs instantaneous communication status. Operation of the framework was demonstrated on the cross-shaped intersection controlled by a CV-data-based adaptive control algorithm.

Implementation of the framework revealed several pitfalls that had to be addressed prior to achieving 
satisfactory performance, namely: (i) The synchronization of shared files" read/write operations has to be of a very high precision. If the final output file is constructed in a series of discrete write operations, it is advised to construct such a file outside of the shared folder until it is complete. (ii) Due to the complexity of the used models, the OMNeT++ simulation of a dense communication network involving a realistic model of a vehicular communications environment is very slow. Even with position updates and message generation frequency set to low values (1s both) and using moderately powerful simulation computer, the run of 2400 simulation seconds can take up to one day of the CPU time, i.e. 24 hours, to finish. For this reason, the framework exploits an optimization method introduced to skip simulation seconds when the simulated traffic control algorithm cannot benefit from the $\mathrm{CV}$ data, e.g. during an interstage, i.e. a predefined safety period between signal phases, where no intervention is possible.

The simulation run time can be further decreased by an implementation of dynamic OMNeT++ node initialization. It is worth noting here that certain protocol models, e.g. IP network layer protocol model, could not be used along with OMNeT++ automatic IP assignment in such a case.

In the near future, the developed framework will be utilized for an investigation of the impact of imperfect communication on the traffic control performance in connected vehicle environment and development of more resilient traffic control algorithms.

\section{Acknowledgement}

This work was supported by the Slovak Research and Development Agency under the contract no. SK-IL-RD-18-005 ICT and smart cars for efficient emergency response and traffic management (SENECA). The authors would like to thank the Israel Ministry of Science, Technology and Space for co-funding this research.

\section{References}

[1] ETSI. EN 302 637-2. Intelligent Transport Systems (ITS); vehicular communications; basic set of applications; part 2: specification of cooperative awareness basic service, 2014.

[2] JING, P., HUANG H., CHEN, L. An adaptive traffic signal control in a connected vehicle environment: a systematic review. Information [online]. 2017, 8(3), 101, [accessed 2020-06-22]. ISSN 2078-2489. Available from: https://doi. org/10.3390/info8030101

[3] MOLISCH, A. F. Wireless communications. 2. ed. Los Angeles, CA: Wiley, 2011. ISBN 978-0-470-74186-3.

[4] GOZALVEZ, J., SEPULCRE M., BAUZA, R. IEEE 802.11p vehicle to infrastructure communications in urban environments. IEEE Communications Magazine [online]. 2012, 50(5), p. 176-183 [accessed 2020-06-22]. ISSN 01636804. Available from: https://doi.org/10.1109/MCOM.2012.6194400

[5] VAN PHU, C. N., FARHI, N., HAJ-SALEM, H., LEBACQUE, J. A vehicle-to-infrastructure communication based algorithm for urban traffic control. In: 5th IEEE International Conference on Models and Technologies for Intelligent Transportation Systems MT-ITS 2017: proceedings [online]. Piscataway, NJ: IEEE, 2017. ISBN 978-1-5090-6484-7. Available from: https://doi.org/10.1109/MTITS.2017.8005594

[6] SAEED, I., ELHADEF, M. Performance evaluation of an IoV-based intersection traffic control approach. In: IEEE International Conference on Internet of Things iThings and IEEE Green Computing and Communications GreenCom and IEEE Cyber, Physical and Social Computing CPSCom and IEEE Smart Data SmartData: proceedings [online]. Piscataway, NJ: IEEE, 2018. ISBN 978-1-5386-7975-3. Available from: https://doi.org/10.1109/Cybermatics_2018.2018.00296

[7] SOMMER, C., GERMAN, R., DRESSLER, F. Bidirectionally coupled network and road traffic simulation for improved IVC analysis. IEEE Transactions on Mobile Computing [online]. 2010, 10(1), p. 3-15 [accessed 2020-06-22]. ISSN 15361233. Available from: https://doi.org/10.1109/TMC.2010.133

[8] PETROV, T., POCTA, P., ROMAN, J., BUZNA, L., DADO, M. A feasibility study of privacy ensuring emergency vehicle approaching warning system. Applied Sciences [online]. 2020, 10(1), 298 [accessed 2020-06-23]. ISSN 2076-3417. Available from: https://doi.org/10.3390/app10010298

[9] PTV GROUP. VISSIM 7: User Manual, 2014.

[10] WEHRLE, K., GUNES M., GROSS, J. Modeling and tools for network simulation. Berlin, Heidelberg: Springer-Verlag, 2010. ISBN 978-3-642-12331-3.

[11] THANH BINH, H., DEY N. Soft computing in wireless sensor networks. Boca Raton: Taylor \& Francis, 2018. ISBN 978-0815395300.

[12] OMNeT++ Discrete Event Simulator [online] [accessed 2019-11-25]. Available from: https://omnetpp.org

[13] SIVAKUMAR, K., DALIN, G. The analysis and implementation of OMNeT++ framework. Global Journal of Computer Science and Technology. 2011, 11(6), p. 65-70. ISSN 0975-4350.

[14] INET Framework [online] [accessed 2019-12-10]. Available from: https://inet.omnetpp.org/

[15] Vehicles in network simulation: the open source vehicular network simulation framework [online] [accessed 2019-1210]. Available from: https://veins.car2x.org

[16] KRAJZEWICZ, D., ERDMANN, J., BEHRISCH, M., BIEKER, L. Recent development and applications of SUMO - 
Simulation of Urban MObility. International Journal on Advances in Systems and Measurements. 2012, 5(3\&4), p. 128-138. ISSN 1942-261x.

[17] IEEE 1609.0-2019. IEEE Guide for Wireless Access in Vehicular Environments (WAVE) Architecture, 2019.

[18] SAIDAllaH, M., El FERGOUGUI, A., ELBELRHITI ELALAOUI, A. A comparative study of urban road traffic simulators. MATEC Web of Conferences [online]. 2016, 81, 05002 [accessed on 2020-06-23]. ISSN 2261-236x. Available from: https://doi.org/10.1051/matecconf/20168105002

[19] KWATIRAYO, S., ALMHANA, J., LIU, Z. Optimizing intersection traffic flow using VANET. In: IEEE International Conference on Sensing, Communications and Networking SECON 2013: proceedings. Piscataway, NJ: IEEE, 2013. ISBN 978-1-4673-1905-8.

[20] IEEE 802.11p-2010. IEEE Standard for information technology - Local and metropolitan area networks - Specific requirements - Part 11: Wireless LAN Medium Access Control (MAC) and Physical Layer (PHY) Specifications Amendment 6: Wireless access in vehicular environment, 2010.

[21] SOMMER, C., JOERER, S., DRESSLER, F. On the applicability of Two-Ray path loss models for vehicular network simulation. In: IEEE Vehicular Networking Conference VNC 2012: proceedings [online]. Piscataway, NJ: IEEE, 2012. ISBN 978-1-4673-4995-6. Available from: https://doi.org/10.1109/VNC.2012.6407446

[22] RAHMAN, A., GBURZYNSKI, B. Hidden problems with the hidden node problem. In: 23rd Biennial Symposium on Communications: proceedings. Piscataway, NJ: IEEE, 2006. ISBN 0-7803-9528-X. 